Trabal, G., Daza, G. y Arboix-Alió, J. (2020). Influencia de las variables contextuales en la intervención del portero de hockey patines en la falta directa. Cuadernos de Psicología del Deporte, 20(2), 139-151

\title{
Influencia de las variables contextuales en la intervención del portero de hockey patines en la falta directa
}

\section{Influence of contextual variables in the intervention of the rink hockey goalkeeper in the free direct hit}

\section{Influência de variáveis contextuais na intervenção do guardaredes de hoquei em patins no livre direto}

\author{
Trabal, G.1, Daza, G.2, Arboix-Alió, J.3 \\ ${ }_{1}$ Department of Physical Activity Sciences, University of Vic (Barcelona, Spain);2 National Institute of \\ Physical Education of Catalonia (INEFC), University of Barcelona (UB) (Barcelona, Spain); зFaculty of \\ Psychology, Education Sciences and Sport Blanquerna, Ramon Llull University (Barcelona, Spain)
}

\section{RESUMEN}

El objetivo del presente estudio fue analizar el efecto de las variables contextuales del partido en la intervención del portero de hockey patines durante las faltas directas. Se realizó un estudio documental basado en la Ok Liga 2015 16 y fueron analizadas todas las faltas directas $(n=637)$. Los datos fueron recogidos de las actas de juego de la Real Federación Española de Patinaje. Se analizaron las variables contextuales: minuto de partido, estado del marcador, momento de la temporada, importancia de la falta directa y localización del partido. Se realizó un análisis correlacional mediante la prueba chi cuadrado de Pearson para identificar la relación entre las variables del estudio y la eficacia del portero. Los resultados constataron que el minuto de partido presenta una relación significativa con la eficacia del portero $(\chi 2=17,665 ;$ Sig. $=, 04 ; p<, 05 ; \mathrm{w}=, 167)$. un aumento de la frecuencia de faltas directas en los momentos finales, el 43,96\% son lanzadas en los últimos 10 minutos de partido, y una mejora de la eficacia del portero en los momentos críticos $(77,1 \%)$. Estos resultados pueden contribuir a una mejor comprensión de la importancia de los porteros en el resultado final de los partidos de hockey patines.

Palabras clave: ventaja de jugar en casa, variables situacionales, tiempo de partido, resultado, competición.

\begin{abstract}
The aim of the present study was to analyze the effect of the match's contextual variables on the roller hockey goalkeeper intervention during free hits. A documentary study based on the Ok Liga 2015-16 was carried out and all the direct free hit of this season were analyzed $(n=637)$. The data were collected from the playing records of the Real Federación Española de Patinaje. The contextual variables analyzed in direct free hit were: the minute of the
\end{abstract}




\section{Trabal, G., Daza, G. y Arboix-Alió, J. (2020)}

match, score status, season time, importance of the direct free hit and match location. A correlational analysis has been performed using the Pearson's chi-squared test to identify the relationship between contextual variables and goalkeeper's efficiency. The results show that the minute of the match has a significant relationship with the goalkeeper's efficiency $(\chi 2=17.665$, Sig. $=.04, \mathrm{p}<.05 ; \mathrm{w}=.167)$, an increase in the frequency of direct free hit in the final moments, $43.96 \%$ are thrown in the last 10 minutes of the game, and an improvement in the goalkeeper's efficiency in critical moments $(77.1 \%)$. These results can contribute to a better understanding of the goalkeepers' importance in roller hockey matches result.

Keywords: home advantage, situational variables, match time, result, competition.

\section{RESUMO}

O objetivo do presente estudo foi analisar o efeito das variáveis contextuais do jogo na intervenção do guarda-redes de hóquei em patins nos livres diretos. Um estudo documental baseado na Ok Liga 2015-16 foi realizado e foram analisados todos os livres diretos $(\mathrm{n}=637)$. Os dados foram coletados das minutas do jogo da Real Federación Española de Patinaje. Foram analisadas as variáveis contextuais: minuto de jogo, estado do marcador, momento da época, importância do livre direto e localização do jogo. Foi realizada um análise correlacional pelo teste quiquadrado de Pearson para identificar a relação entre as variáveis do estudo e a eficácia do guarda-redes. Os resultados mostraram que o minuto de jogo tem relação significativa com a eficácia de guarda-redes $(\chi 2=17.665$, $\operatorname{Sig}=.04, p<.05 ; \mathrm{w}=, 167), \mathrm{um}$ aumento na frequiência dos livres diretos nos momentos finais do jogo, $43.96 \%$ são marcados nos últimos 10 minutos do jogo, e uma melhora na eficácia do guarda-redes nos momentos críticos (77.1\%). Esses resultados podem contribuir para uma melhor compreensão da importância dos guarda-redes no resultado final dos jogos de hóquei em patins.

Palavras chave: vantagem de campo, variáveis situacionais, tempo de jogo, resultado, competição.

\section{INTRODUCCIÓN}

En la temporada 2009-10 entró en vigor el nuevo reglamento en el hockey patines (HP) que generó modificaciones importantes en la falta directa (FD): la forma de ejecución y las infracciones que originan una FD (Rink Hockey Committee, 2009). La gran importancia de estas acciones en el HP viene justificada porque en muchos partidos $y$ competiciones la victoria o la derrota puede ser decidida por la eficacia en estos lanzamientos (Pauls, 2012; Trabal, 2019; Trabal, Daza y Riera, 2019a). Siendo la FD una acción de juego en la que se crea una relación de oposición entre portero y jugador con influencia recíproca (Navia y Ruiz, 2014) y en la cual se establece entre ellos un duelo asimétrico en el que el jugador tiene el objetivo de marcar gol y el portero de evitarlo (Folguera, 2000; Trabal, Daza y Riera, 2019b).

El análisis del rendimiento del HP pone de manifiesto dos aspectos importantes del portero. En primer lugar, su importancia es valorada por encima del 50\% del equipo y esto conlleva que sus actuaciones pueden tener una implicación directa en el rendimiento final de los equipos (Folguera, 2000; Trabal, 2016; Trabal, Daza y Riera, 2020). El segundo, que el rendimiento en el HP y del portero es multifactorial (Pauls, 2012; Trabal, 2019; Vaz, 2011), estando determinado por diferentes variables técnicas, tácticas, psicológicas y condicionales.

Aunque Pauls (2012) afirma que las variables contextuales pueden ser factores que influyen en el rendimiento del HP, son pocos los estudios que han analizado la influencia que estas tienen en el resultado final en este deporte. La localización del partido ha sido analizada en la Ok Liga (España) y se demuestra que los equipos locales obtienen un porcentaje superior de puntos jugando como anfitriones, home advantage (Arboix-Alió, AguileraCastells, Buscà, Sánchez-López y García-Eroles, 2019; Arboix-Alió, Buscà, Trabal, Aguilera-Castells y Sánchez-López, 2020), el 59,8\% según ArboixAlió y Aguilera-Castells (2019) y el 58,32\% según Gómez, Pollard y Luis-Pascual (2011). Se observa que marcar el primer gol es una variable asociada a la victoria final, existiendo la ventaja de marcar primero en la Ok Liga con un efecto del 64,14 y en la Primera División Nacional del 62,91\% (Arboix-Alió y 


\section{Variables contextuales para el portero de hockey patines faltas directas}

Aguilera-Castells, 2018). Otra variable contextual que ha mostrado ser un valor predictivo de victoria en dicho deporte es el hecho de llegar a la media parte con el marcador favorable (Arboix-Alió et al., 2019).

En otros deportes colectivos los factores contextuales han sido objeto de estudio para determinar si estas variables pueden ser predictores del resultado final, abordando la localización del partido, la diferencia en el marcador, el minuto de partido, el momento de la temporada o la importancia del partido (MorilloBaro, Reigal y Hernández-Mendo, 2015; VázquezDiz, Morillo-Baro, Reigal, Morales-Sánchez y Hernández-Mendo, 2019).

Las investigaciones que han estudiado la localización del partido home advantage muestran una mayor cantidad de victorias de los equipos locales en deportes como el fútbol (Pollard, 1986; Pollard y Gómez, 2009), baloncesto (Gómez y Pollard, 2011), balonmano (Gutiérrez, Saavedra y FernándezRomero, 2012), hockey hielo (Hoffmann, Loughead, Dixon y Crozier, 2017) y rugby (Adams y Kupper, 1994). Otras investigaciones realizadas en deportes colectivos como básquet o béisbol muestran que la ventaja de jugar en casa tiende a desaparecer cuando los partidos son considerados decisivos (Baumeister y Steinhilber, 1984; Schlenker, Phillips, Boniecki, y Schlenker, 1995). Investigaciones sobre la eficacia de las acciones técnicas en relación con la condición de local o visitante muestran que no existen relaciones significativas (Casimiro, 2010; Hantau y Hantau, 2014; Hoffmann et al., 2017; Jiménez-Torres y López-Gutiérrez, 2012; Liardi y Carron, 2011). En el caso de hockey hielo (Hoffmann et al., 2017; Liardi y Carron, 2011) se ha constatado que los equipos locales ganan más partidos que los visitantes, pero cuando los partidos se definen en un desempate en los penaltis (shootout), esta tendencia del equipo local disminuye. En baloncesto, la eficacia de los tiros libres manifiesta diferencias poco significativas a favor de los equipos locales (Jiménez-Torres y López-Gutiérrez, 2012) y en balonmano se observa una mayor eficacia de los porteros cuando estos actúan como visitantes (Casimiro, 2010; Hantau y Hantau, 2014).

El estado del marcador también ha sido una variable contextual investigada relacionada con la eficacia en las acciones de los deportistas. En baloncesto, aunque no existan diferencias significativas en la eficacia en los tiros libres en función del estado del marcador (Jiménez-Torres y López-Gutierrez, 2012; Whitehead, Butz, Kozar y Vaughn, 1996), sí que hay alteraciones de la eficacia de estos lanzamientos en relación con el resultado final del partido. Según Jiménez-Torres y López-Gutiérrez (2012) y Ahart (1973) la condición óptima para alcanzar el mejor porcentaje de acierto es cuando el jugador se encuentra ante situaciones de presión media, con resultados favorables entre los cinco y los 10 puntos. Por el contrario, Buceta (1992) y Labrador, Crespo, Buceta y González (1995) afirmaron que el máximo rendimiento se consigue en situaciones de máxima presión, cuando el resultado del partido está muy igualado.

La eficacia de las habilidades en los diferentes momentos del partido es otra variable contextual analizada. En el futbol se constató que en los en los últimos 15 minutos de partido se marcan más goles a balón parado (Castellano y Zubillaga, 1995a, 1995b, 1995c) y se lanzan la mayor cantidad de lanzamientos de faltas (Maneiro, 2014). En el balonmano Casimiro (2010) demostró una relación significativa entre el tiempo de partido y la eficacia del portero, siendo los últimos 10 minutos de partido el momento en el que estos obtienen la eficacia más baja. En baloncesto, a medida que avanza el partido aumenta la cantidad de lanzamientos de tiro libres (Jiménez-Torres y López-Gutiérrez, 2012; Kozar, Vaughn, Whitfield y Lord, 1994) y disminuye la eficacia (Labrador et al., 1995).

De la relación entre el tiempo de partido y el estado del marcador surge el concepto de momento crítico, definido como el momento clave que contribuye decisivamente en las victorias o en las derrotas (Navarro, Gómez, Lorenzo, Lorenzo y Jiménez, 2015). En esta línea, McEwan, Gini, y Bray (2012) analizaron los shootout en hockey hielo y observaron que los porteros que actúan como locales tienen un porcentaje de eficacia (\%EP) más alto que los porteros visitantes cuando tienen que defender un penalti para no perder el partido. Por el contrario, el $\%$ EP de los locales es inferior respecto a los visitantes cuando el penalti a detener es para ganar el partido. Jiménez-Torres y López-Gutiérrez (2012) detectaron que, en las prórrogas de baloncesto, y en comparación con las segundas partes de partido, los jugadores locales bajan su acierto en los tiros libres y 
Trabal, G., Daza, G. y Arboix-Alió, J. (2020)

los jugadores visitantes lo aumentan. Roffé, De la Vega, Garcia-Mas y Llinás (2007) determinaron que los "goles psicológicos" en fútbol, anotados en el último tramo de la primera parte, influyen positivamente en el resultado final del equipo que marca este gol. Teniendo en cuenta las características anteriores, la enorme importancia y responsabilidad que el HP otorga al portero en el rendimiento final de los equipos, la existencia de diversas variables influyentes en el HP y la falta de investigaciones en el estudio de la FD y del rendimiento de los porteros en el HP, se plantea la necesidad de estudiar dicha circunstancia. Así pues, el objetivo del presente estudio fue analizar la influencia de las siguientes variables contextuales de partido: a) localización, b) minuto de partido, c) estado del marcador, d) momento de la temporada, e) importancia de la falta directa; sobre la eficacia del portero en las FD.

\section{MATERIAL Y MÉTODOS}

\section{Participantes}

Se realizó un estudio documental basado en la $\mathrm{Ok}$ Liga 2015-16.

Tabla 1. Criterios, categorías y descripción de las categorías utilizadas para analizar el contexto.

\begin{tabular}{|c|c|c|}
\hline Criterio & Categoría & Descripción categoría \\
\hline Localización del partido & $\begin{array}{c}\text { Local } \\
\text { Visitante } \\
\end{array}$ & $\begin{array}{c}\text { El equipo del portero juega de local } \\
\text { El equipo del portero juega de visitante }\end{array}$ \\
\hline Minuto de partido I & $\begin{array}{l}\text { PP1 } \\
\text { PP2 } \\
\text { PP3 } \\
\text { PP4 } \\
\text { PP5 } \\
\text { SP1 } \\
\text { SP2 } \\
\text { SP3 } \\
\text { SP4 } \\
\text { SP5 } \\
\end{array}$ & $\begin{array}{c}0 \text { min }-5 \text { min } \\
5 \text { min } 1 \mathrm{~s}-10 \mathrm{~min} \\
10 \min 1 \mathrm{~s}-15 \mathrm{~min} \\
15 \min 1 \mathrm{~s}-20 \mathrm{~min} \\
20 \min 1 \mathrm{~s}-25 \mathrm{~min} \\
25 \min 1 \mathrm{~s}-30 \mathrm{~min} \\
30 \min 1 \mathrm{~s}-35 \mathrm{~min} \\
35 \min 1 \mathrm{~s}-40 \mathrm{~min} \\
40 \min 1 \mathrm{~s}-45 \mathrm{~min} \\
45 \min 1 \mathrm{~s}-50 \mathrm{~min}\end{array}$ \\
\hline Minuto de partido II & $\begin{array}{l}\text { Primera parte } \\
\text { Segunda parte }\end{array}$ & $\begin{array}{c}0 \min -25 \min \\
25 \min 1 \mathrm{~s}-50 \min \\
\end{array}$ \\
\hline $\begin{array}{l}\text { Estado en el marcador } \\
\text { (resultado del equipo del portero } \\
\text { en el momento que se realiza el } \\
\text { lanzamiento de FD) }\end{array}$ & $\begin{array}{l}\text { EMP } \\
\text { V1G } \\
\text { V2G } \\
V+2 G \\
D 1 G \\
D 2 G \\
D+2 G\end{array}$ & $\begin{array}{c}\text { Empate } \\
\text { Victoria de un gol } \\
\text { Victoria de dos goles } \\
\text { Victoria de más de dos goles } \\
\text { Derrota de un gol } \\
\text { Derrota de dos goles } \\
\text { Derrota de más de dos goles }\end{array}$ \\
\hline Momento de la temporada & $\begin{array}{l}1 \mathrm{~V} \\
2 \mathrm{~V} \\
\end{array}$ & $\begin{array}{l}\text { Primera vuelta } \\
\text { Segunda vuelta }\end{array}$ \\
\hline Importancia de la FD & $\begin{array}{l}\text { IMP } \\
\text { NIMP }\end{array}$ & $\begin{array}{l}\text { Importante: la FD se lanza en los últimos cinco minutos } \\
\text { con resultado igualado (EMP, V1G o D1G) } \\
\text { No importante: la FD no se lanza en los últimos cinco } \\
\text { minutos con resultado igualado (EMP, V1G o D1G) }\end{array}$ \\
\hline Eficacia del portero & $\begin{array}{l}\text { GOL } \\
\text { NGOL }\end{array}$ & $\begin{array}{l}\text { El lanzamiento de FD es gol. } \\
\text { El lanzamiento de FD no es gol. }\end{array}$ \\
\hline
\end{tabular}

La muestra seleccionada fue intencional o por conveniencia (Anguera et al., 1995). Se seleccionaron todas las FD ejecutadas en la Ok Liga de la temporada 2015-16 (en total 650). Finalmente fueron analizadas 637 , descartando 13 FD por falta de datos.
El estudio es fiel a los principios éticos de toda investigación científica y se han seguido los requisitos establecidos por la American Phychological Association (American Psycological Association, 2002). 


\section{Variables contextuales para el portero de hockey patines faltas directas}

\section{Instrumentos}

Se utilizó la base de datos de la Federación Española de Patinaje para obtener la información de las FD a través de las actas electrónicas de juego y se utilizó una plantilla Excel como instrumento de registro. Se creó un instrumento de observación ad hoc teniendo en cuenta las variables contextuales y la eficacia en la FD: minuto de partido, estado del marcador, momento de la temporada, importancia de la FD, localización del partido y eficacia del portero. (Tabla 1). El porcentaje de eficacia (\%EP) quedó acotado por el porcentaje de FD que no terminan en gol.

\section{Procedimiento y Análisis estadístico}

Mediante el instrumento de registro se han recogido los datos de cada FD. Dicho instrumento fue previamente validado por un grupo de expertos considerándose adecuado para el análisis de las variables contextuales en el HP, con acuerdos del $100 \%$. Seguidamente, los datos se han volcado al programa SPSS v.23 para realizar el análisis estadístico. Se ha realizado un primer análisis descriptivo para presentar la frecuencia de FD sancionadas en relación a cada variable del estudio. Posteriormente, un análisis correlacional mediante la prueba Chi-Cuadrado de Pearson para identificar la relación entre las variables del estudio y el resultado de la finalización: gol / no gol. Adicionalmente se calculó el tamaño del efecto para las variables que presentaron significación. El criterio para la significación fue $p<, 05$. Mediante el coeficiente de contingencia se ha analizado la intensidad de la relación entre variables.

Con el fin de analizar la fiabilidad de los datos obtenidos de las estadísticas oficiales de la Ok Liga, se realizó un análisis observacional por dos observadores expertos. Se utilizó la metodología observacional por las posibilidades que ofrece para el análisis de la interacción portero-jugador en su contexto y dinámica habitual (Anguera y HernándezMendo, 2013, 2014) y por su utilidad demostrada en el HP (Hernández-Mendo y Anguera, 2002). Los observadores estaban en posesión de la titulación de Entrenador Nacional de Hockey Patines y de Ciencias de la Actividad Física y el Deporte. Los observadores siguieron las pautas de preparación propuestas Anguera (1992). Se analizaron 100 FD teniendo en cuenta las variables propuestas en la investigación (tabla 1). Los resultados de las pruebas obtuvieron unos resultados de concordancia intra observador $(k=, 992)$ e inter observador $(k=, 984)$, asegurando la fiabilidad del instrumento.

Además, se ha realizado un análisis de Generalizabilidad (Cronbach, Gleser, Nanda y Rajaratnam, 1972), mediante el software SAGT, versión, 1.0 (Hernández-Mendo, Blanco-Villaseñor, Pastrana, Morales-Sánchez y Ramos-Pérez (2016) (Tabla 2). Al modo de Blanco-Villaseñor, Castellano, Hernández-Mendo, Sánchez-López y Usabiaga (2014), se han realizado dos planes de medida para abordar: a) la generalizabilidad de los resultados obtenidos (número de faltas directas que componen el muestreo) y b) la validez del instrumento de observación: a) El coeficiente de generalizabilidad (relativo y absoluto $=0,996$ ) correspondiente al plan de medida [Categorías] / [Faltas Directas] establece que con el número de Faltas Directas analizadas se obtiene una elevada fiabilidad de precisión de generalización. b) Respecto al plan de medida [Faltas Directas] / [Categorías] el coeficiente de generalizabilidad (relativo y absoluto $=0,000$ ), avala en el marco teórico de la Teoría de la Generalizabilidad- la validez del instrumento de observación diseñado (Blanco-Villaseñor y EscolanoPérez, 2017; Blanco-Villaseñor, Castellano, Hernández-Mendo, Sánchez-López y Usabiaga, 2014).

\section{RESULTADOS}

\section{FD lanzadas y goles marcados}

Del total de las FD analizadas, a medida que avanza el partido, sobre todo en la segunda parte, aumentaron las FD señaladas (figura 1), evolucionando de las cuatro FD $(0,6 \%)$ señaladas en los primeros cinco minutos de partido (PP1) hasta las 169 FD (26,5\%) en los últimos cinco minutos (SP5). En las segundas partes se lanzaron el $82,74 \%$ de las FD y se marcaron el $84,12 \%$ de los goles de FD (tabla 3). 
Trabal, G., Daza, G. y Arboix-Alió, J. (2020)

Tabla 2. Resultados correspondientes al diseño de generalizabilidad [Categorías] [Faltas Directas]

\begin{tabular}{lllllllll}
\hline $\begin{array}{l}\text { Fuentes de } \\
\text { variación }\end{array}$ & $\begin{array}{l}\text { Suma de } \\
\text { cuadrados }\end{array}$ & $\begin{array}{l}\text { Grado de } \\
\text { libertad }\end{array}$ & $\begin{array}{l}\text { Cuadrado } \\
\text { medio }\end{array}$ & Aleatorio & Mixtos & Corregidos & \% & $\begin{array}{l}\text { Error } \\
\text { estándar }\end{array}$ \\
\hline [fd] & 0,33 & 636 & 0,001 & $-0,005$ & $-0,005$ & $-0,005$ & 0 & 0 \\
[cat] & 1014,638 & 26 & 39,025 & 0,061 & 0,061 & 0,061 & 30,645 & 0,016 \\
[fd][cat] & 2284,621 & 16536 & 0,138 & 0,138 & 0,138 & 0,138 & 69,355 & 0,002 \\
\hline
\end{tabular}

\section{PORCENTAJE DE FD \\ SEÑALADAS POR MINUTO DE PARTIDO}

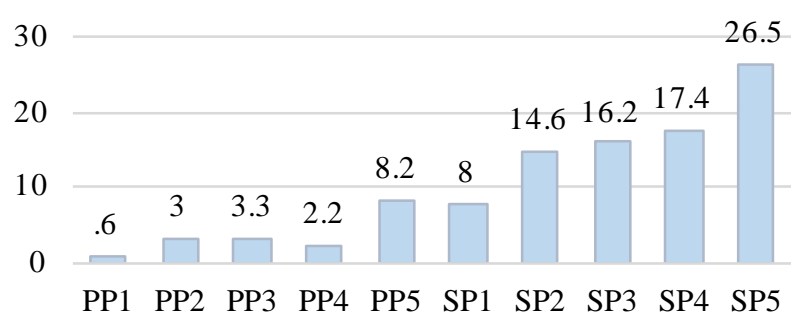

Figura 1. Evolución del porcentaje de FD señaladas en relación con el minuto de partido.

Tabla 3. FD lanzadas, goles marcados de FD y \%EP en las FD en relación a las variables contextuales.

\begin{tabular}{|c|c|c|c|c|}
\hline Criterio & Categoría & FD lanzadas & Goles de FD & $\%$ EP \\
\hline \multirow[t]{10}{*}{ Minuto del partido I } & $\mathrm{PP} 1: 0 \mathrm{~min}-5 \mathrm{~min}$ & 4 & 0 & 100 \\
\hline & $\mathrm{PP} 2: 5 \min 1 \mathrm{~s}-10 \mathrm{~min}$ & 19 & 10 & 47,4 \\
\hline & PP3: $10 \min 1 \mathrm{~s}-15 \min$ & 21 & 5 & 76,2 \\
\hline & PP4: $15 \min 1 \mathrm{~s}-20 \mathrm{~min}$ & 14 & 3 & 78,6 \\
\hline & PP5: $20 \min 1 \mathrm{~s}-25 \mathrm{~min}$ & 52 & 12 & 76,9 \\
\hline & $\mathrm{SP} 1: 25 \min 1 \mathrm{~s}-30 \mathrm{~min}$ & 51 & 13 & 74,5 \\
\hline & $\mathrm{SP} 2: 30 \min 1 \mathrm{~s}-35 \mathrm{~min}$ & 93 & 32 & 65,6 \\
\hline & SP3: $35 \min 1 \mathrm{~s}-40 \mathrm{~min}$ & 103 & 38 & 63,1 \\
\hline & $\mathrm{SP} 4: 40 \min 1 \mathrm{~s}-45 \mathrm{~min}$ & 111 & 38 & 65,8 \\
\hline & SP5: $45 \min 1 \mathrm{~s}-50 \mathrm{~min}$ & 169 & 38 & 77,5 \\
\hline \multirow[t]{2}{*}{ Minuto de partido II } & Primera parte: $0 \mathrm{~min}-25 \mathrm{~min}$ & 110 & 30 & 72,7 \\
\hline & Segunda parte: $25 \min 1 \mathrm{~s}-50 \mathrm{~min}$ & 527 & 159 & 69,8 \\
\hline \multirow[t]{2}{*}{ Localización del partido } & Local & 334 & 99 & 70,3 \\
\hline & Visitante & 303 & 90 & 70,4 \\
\hline \multirow[t]{2}{*}{ Momento de la temporada } & Primera vuelta & 321 & 102 & 68,2 \\
\hline & Segunda vuelta & 316 & 87 & 72,5 \\
\hline \multirow[t]{7}{*}{ Estado del marcador } & Empate & 110 & 34 & 69,1 \\
\hline & Victoria de un gol & 108 & 31 & 71,3 \\
\hline & Victoria de dos goles & 59 & 17 & 71,2 \\
\hline & Victoria de más de dos goles & 91 & 25 & 72,5 \\
\hline & Derrota de un gol & 113 & 31 & 72,6 \\
\hline & Derrota de dos goles & 90 & 28 & 68,9 \\
\hline & Derrota de más de dos goles & 66 & 23 & 65,2 \\
\hline \multirow[t]{2}{*}{ Immortancia de ta FD } & Importante & 70 & 16 & 77,1 \\
\hline & No importante & 567. & 173 & 69,5 \\
\hline
\end{tabular}




\section{Variables contextuales para el portero de hockey patines faltas directas}

En relación a la localización del partido se observa que los equipos que jugaron como locales lanzaron más FD $(52,43 \%)$ que los equipos que actuaron como visitantes (47,57\%). Al mismo tiempo, los equipos locales marcaron más goles de FD, un 52,38\%, que los equipos visitantes, un 47,61.

Los resultados de la influencia del momento de la temporada sobre las FD permiten observar que en la primera vuelta se lanzaron $321 \mathrm{FD}(50,39 \%)$ y en la segunda vuelta $316 \mathrm{FD}(49,61 \%)$. En la primera parte del campeonato se marcaron el $53,97 \%$ de goles de FD mientras que en la segunda parte el 46,03\%. Cuando el estado del marcador presenta resultados igualados (empate y victoria o derrota de un gol) se lanzaron el 51,96\% de las FD. El 40,5\% de FD que recibió el portero fueron con el resultado favorable, mientras que el 42,23\% de FD las recibió estando con un resultado desfavorable. Los resultados del estado del marcador, presentados en la tabla 4, diferencian si el portero actúa como local o visitante y permiten destacar que los locales afrontaron más FD en situación de resultado favorable $(47,9 \%$ que los porteros visitantes $(33,8 \%)$.

Tabla 4. FD señaladas, porcentaje de FD señaladas y \%EP en relación al estado del marcador y a condición de local o visitante del portero.

\begin{tabular}{ccccccc}
\hline \multirow{2}{*}{$\begin{array}{c}\text { Estado del } \\
\text { marcador }\end{array}$} & \multicolumn{3}{c}{ Portero local } & \multicolumn{3}{c}{ Portero visitante } \\
\cline { 2 - 7 } & FD & \%FD & \%EP & FD & \%FD & \%EP \\
\hline Empate & 52 & 17,2 & 71,2 & 58 & 17,4 & 67,2 \\
Victoria & 145 & 47,9 & 69,7 & 13 & 33,8 & 74,3 \\
Derrota & 106 & 35 & 74,5 & 163 & 48,8 & 68,7 \\
\hline Total & 303 & 100 & & 334 & 100 & \\
\hline
\end{tabular}

En los momentos de máxima importancia del partido se marcaron el $8,5 \%$ de los goles de FD y el portero tuvo que afrontar el 10,9\% de FD. Para los porteros locales el 9,9\% de FD lanzadas en contra se encuentran dentro de esta fase de partido mientras que, para los porteros visitantes, el 12\% de FD fueron ejecutadas en estos momentos decisivos del partido.

\section{Eficacia del portero en la FD}

En la tabla 5 se presentan los resultados de las correlaciones entre la eficacia del portero en la FD y las diferentes variables contextuales. La variable minuto de partido, analizada teniendo en cuenta el tiempo de partido dividido por 10 periodos de cinco minutos, es la única variable contextual que tuvo una relación significativa con la eficacia del portero en la FD $(\mathrm{p}=, 04 ; \mathrm{w}=, 167) . \mathrm{El}$ coeficiente de contingencia de 0,16 refleja una asociación débil entre la variable minuto de partido y la efectividad.

Tabla 5. Resultados de las pruebas de chi cuadrado de Pearson y coeficiente de contingencia.

\begin{tabular}{lcccc}
\hline Criterio & $\chi^{\mathbf{2}}$ & Sig. & CC & ES \\
\hline $\begin{array}{l}\text { Minuto de partido I (dividido } \\
\text { en 10 períodos de tiempo) }\end{array}$ & $\mathbf{1 7 , 6 6}$ & $\mathbf{, 0 4} *$ & $\mathbf{, 1 6}$ & $\mathbf{, 1 6 7}$ \\
$\begin{array}{l}\text { Minuto de partido II (1 }{ }^{\mathrm{a}} \text { y } 2^{\mathrm{a}} \\
\text { parte) }\end{array}$ & 0,36 &, 54 & & \\
Localización del partido &, 000 &, 98 & & \\
$\begin{array}{l}\text { Momento de la temporada (1 } \\
\text { y 2 } 2^{\mathrm{a}} \text { vuelta) }\end{array}$ & 1,37 &, 24 & & \\
Estado del marcador & 1,56 &, 95 & \\
Importancia de la FD & 1,74 &, 18 & \\
\hline
\end{tabular}

En relación con la variable minuto de partido se observa que en SP5, período en el que se lanzaron más del $25 \%$ de FD, el portero obtuvo un \%EP del $77,5 \%$. Este valor se encuentra por encima del promedio de \%EP en la FD que es del 70,3\%. La eficacia del portero en las primeras partes fue del $72,7 \%$, superior a la eficacia de las segundas partes que fue del 69,8\%. El \%EP en relación a la localización del partido permitió observar como los porteros locales tuvieron una eficacia del 70,3\% mientras que los visitantes del 70,4\%. La eficacia de los porteros también presentó valores distintos en relación al momento de la temporada, evolucionando de un \%EP del 68,2\% en la primera vuelta hasta un $\%$ EP del $72,5 \%$ en la segunda vuelta.

El estado del marcador también generó \%EP fluctuante, los más altos se obtuvieron cuando el portero tuvo que defender una FD con $\mathrm{V}+2 \mathrm{G}$, con un $72,5 \%$, y en un $72,6 \%$ con D1G. Por el contrario, los $\%$ EP más bajos se consiguieron cuando el portero tuvo que intervenir en el lanzamiento de FD con 


\section{Trabal, G., Daza, G. y Arboix-Alió, J. (2020)}

$\mathrm{D} 2 \mathrm{G}, 68,9 \%$, o de $\mathrm{D}+2 \mathrm{G}, 65,2 \%$. Los \%EP en función de si recibe el lanzamiento de FD con un resultado favorable o desfavorable muestran una eficacia superior cuando el portero tuvo que defender la FD con victoria, siendo esta del $71,7 \%$, por un $69,51 \%$ cuando el resultado es desfavorable.

Esta relación entre estado del marcador y \%EP, concretada en función de si el portero juega como local o visitante, manifestó que la eficacia de estos, tanto de los locales $(\chi 2=, 058$; Sig. $=, 972 ; \mathrm{p}>, 05)$, como la de los visitantes $(\chi 2=1,340$; Sig. $=, 512$; $p>, 05)$, no presentó relación significativa con el estado del marcador en el momento de recibir la FD. Sin embargo, sí se observaron diferencias en el \%EP en función del estado del marcador y de la localización del partido. El \%EP más alto de los porteros locales, en relación con el resultado, se consiguió cuando estos interactuaron en la FD con derrota $(74,5 \%)$. Por su parte, los porteros visitantes alcanzaron el \%EP más alto cuando su actuación en la FD fue con el resultado a favor $(74,3 \%)$.

Los resultados de la eficacia del portero en relación con la importancia de la FD evidenciaron que en los momentos de máxima importancia el \%EP fue del $77,1 \%$. Por su parte, en los momentos que no son considerados de importancia, la eficacia fue del $69,5 \%$. Entre porteros locales y visitantes, y en correspondencia con estos momentos críticos, el \%EP más alto que se consiguió alcanzó el 83,3\% por parte de los porteros locales (tabla 6).

Tabla 6. FD señaladas, $\%$ de FD señaladas y $\%$ EP en relación a la importancia y a la condición de local o visitante del portero.

\begin{tabular}{|c|c|c|c|c|c|c|}
\hline \multirow{2}{*}{$\begin{array}{l}\text { Importanci } \\
\text { a de la FD }\end{array}$} & \multicolumn{3}{|c|}{ Portero local } & \multicolumn{3}{|c|}{ Portero visitante } \\
\hline & FD & $\begin{array}{c}\% \mathrm{~F} \\
\mathrm{D}\end{array}$ & $\%$ ЕP & $\overline{\text { FD }}$ & $\% \mathrm{FD}$ & $\% \mathrm{EP}$ \\
\hline Importante & 30 & 9,9 & 83,3 & 40 & 12 & 72,5 \\
\hline $\begin{array}{c}\text { No } \\
\text { importante }\end{array}$ & 273 & 90,1 & 68,9 & 294 & 88 & 70,1 \\
\hline Total & 303 & 100 & & 334 & 100 & \\
\hline
\end{tabular}

\section{DISCUSIÓN}

El objetivo de la presente investigación fue analizar la influencia de las variables contextuales de partido en la intervención del portero de HP durante las FD. De las distintas variables contextuales analizadas: a) localización, b) minuto de partido, c) estado del marcador, d) momento de la temporada, e) importancia de la falta directa; el minuto de partido fue la única influyente en el rendimiento del portero.

El aumento de la cantidad de FD lanzadas a medida que avanza el partido, siendo el SP5 el período en el que se concentran la mayor cantidad de FD, se justifica por la normativa que regula las FD y por la estrategia de los equipos. Cuando un equipo comete 10 faltas de equipo es castigado con una FD en contra, seguidamente a esta primera acumulación de 10 faltas, cada cinco faltas de equipo este es sancionado con una nueva FD. Por consecuencia, a medida que avanza el partido los equipos acumulan faltas de equipo y estas son sancionadas con FD. Estratégicamente, muchos equipos esperan a los instantes finales de los partidos para realizar un juego más intenso, ya sea para presionar al equipo rival para recuperar la desventaja en el marcador o para mantener un resultado favorable. Este aumento de la intensidad genera situaciones que facilitan cometer faltas de equipo o recibir tarjetas azules, siendo esta última amonestación sancionada directamente con una FD. En comparación con otras investigaciones de otros deportes de cooperación y oposición que han analizado la influencia del tiempo de partido, se observa el aumento de faltas en los minutos finales de partido (Castellano y Zubillaga, 1995a, 1995b, 1995c; Jiménez-Torres y López-Gutiérrez, 2012; Maneiro, 2014; Kozar et al., 1994) coincidiendo con los datos que se muestran sobre las FD.

El presente estudio ha constatado que no existe relación significativa entre la localización del partido y la efectividad del portero en las FD. Esta falta de relación significativa corrobora los resultados en otros deportes que exponen la falta de influencia de la condición de local o visitante con la eficacia de acciones específicas a pelota parada (Casimiro, 2010; 2011; Gayton, Perry, Loignon y Ricker, 2011; Gómez et al., 2014; Loignon, Gayton, Brown, Steinroeder y Johnson, 2007; Jiménez-Torres y López-Gutiérrez, 2012). La explicación del porqué es más frecuente observar influencia de la localización del partido con la victoria o derrota en partidos de deportes colectivos (Courneya y Carron, 1992; Johnston, 2008; Legaz, Moliner y Munguía, 2013; 


\section{Variables contextuales para el portero de hockey patines faltas directas}

Pollard, 1986) y no tanto en la influencia de la condición de local o visitante respecto acciones concretas y aisladas dentro el juego: tiros libres, FD o penaltis, puede ser justificada por la argumentación expuesta por Casimiro (2010) en el balonmano. Según este autor, las acciones como la FD son interacciones en las que sólo están involucrados el portero y el lanzador, es decir, se trata de acciones aisladas en un contexto diferente del resto de acciones en el transcurso de un partido con relaciones de cooperación y oposición. Esta situación aislada conlleva que para el portero sea más fácil centrar en su campo visual los aspectos importantes a atender para obtener la información necesaria para determinar la trayectoria del lanzamiento y los movimientos del jugador. En estas acciones es más fácil reducir la atención de los aspectos externos que pueden influenciar al deportista debido a la localización del partido, como puede ser el público, el desconocimiento del espacio, la falta adaptación a la pista o la influencia del arbitraje (Myers, 2014; Myers y Balmer, 2012; Sánchez et al., 2001; Smith, 2005).

La relación entre el estado del marcador y el \%EP, aunque no existen diferencias significativas, muestra que el \%EP más alto se alcanza cuando el portero tiene que defender una FD con $\mathrm{V}+2 \mathrm{G}$, una situación que se podría considerar de presión media (Ahart, 1973), y con D1G, que es un momento de presión máxima debido a que si el portero no evita el gol se abre una brecha importante en el marcador. Esta situación de presión máxima puede aumentar la motivación del portero para estimular su esfuerzo y mejorar su disponibilidad de recursos y estrategias para mejorar el rendimiento (Wilson, Vine y Wood, 2009). En referencia a los \%EP más bajos estos se obtienen cuando el portero tiene que afrontar la FD con $\mathrm{D} 2 \mathrm{G}$ o $\mathrm{D}+2 \mathrm{G}$. Con estos datos se puede afirmar que cuando el portero se encuentra ante una situación de resultado muy adversa disminuye su prestación, puede ser por sentir una gran presión de no seguir recibiendo goles y porqué el equipo rival se puede distanciar mucho en el marcador o, todo lo contrario, que el portero no afronte la FD con la misma motivación debido al resultado desfavorable y a la poca importancia que puede tener defender o no la FD.
En las situaciones críticas y de máxima importancia los porteros que actúan como locales mejoran su rendimiento respecto a los porteros visitantes. Esta mejora observada en los porteros locales también se manifiesta en los porteros de hockey hielo en los shootout (McEwan et al., 2012; Tauer, Guenther y Rozek, 2009). Contrariamente, nuestros resultados se oponen a los de Jiménez-Torres y López-Gutiérrez (2012) en los tiros libres de baloncesto en las prórrogas, los cuales afirman que son los visitantes quienes aumentan el rendimiento en estos momentos críticos. La mejora del rendimiento de los porteros locales en estos momentos importantes puede ser explicado por la mejora de los estados psicológicos mediante el aprovechamiento de las ventajas de jugar como local: la presencia de los aficionados, el conocimiento y familiarización con la pista o el terreno de juego, la ausencia de viajes para jugar, la aplicación de las normas del arbitraje y la responsabilidad de representar la territorialidad (Carron, Loughhead, y Bray, 2005; Courneya y Carron, 1992; Nevill y Holder, 1999; Pollard, 1986; 2006). Este momento crítico, junto a los factores contextuales a los que está inmerso el portero visitante, pueden influir negativamente y alterar el estado de concentración que dificulta mantener la atención sobre los estímulos pertinentes.

\section{CONCLUSIONES}

Los resultados de la investigación confirman que únicamente el factor contextual minuto de partido tiene una relación estadísticamente significativa con la eficacia del portero en las FD. Se destaca la importancia del portero en los momentos finales de los partidos: incremento de la cantidad de FD señaladas y aumento del rendimiento de los porteros en los momentos críticos, especialmente por parte de los porteros locales.

\section{APLICACIONES PRÁCTICAS}

Estos resultados pueden contribuir a una mejor comprensión de la importancia de los porteros en el resultado final de los partidos de hockey patines. Este hecho, justifica y motiva el interés en realizar el análisis de los factores contextuales en relación con el portero teniendo en cuenta la importancia de los partidos, el nivel de los equipos que se enfrentan y la influencia de la localización diferenciando entre los equipos locales que consiguen crear una atmósfera de 


\section{Trabal, G., Daza, G. y Arboix-Alió, J. (2020)}

gran presión sobre los equipos visitantes respecto aquellos que juegan de locales en ambientes tranquilos. El conocimiento de las variables contextuales que afectan la eficacia del portero puede ser de gran interés para los entrenadores en la creación de situaciones de entrenamiento específicas para la mejora del rendimiento de los porteros, así como para aplicar las estrategias psicológicas adecuadas para obtener el rendimiento óptimo. Del mismo modo, también puede ser útil para los entrenadores para planificar la estrategia a seguir por sus equipos en relación con los diferentes momentos del partido.

\section{BECAS, AYUDAS O SOPORTE FINANCIERO}

El estudio ha contado con recursos del Grupo de Investigación Social y Educativa de la Actividad Física y el Deporte (SGR 1162), Institut Nacional d'Educació Física de Catalunya (INEFC), Universitat de Barcelona (UB).

\section{REFERENCIAS}

1. Adams, R., y Kupper, S. (1994). The effect of expertise on peak performance: the case of homefield advantage. Journal of Sport Behavior, 17, 108-119.

2. Ahart, F. C. (1973). The effect of score differential on basketball free throw shooting. Ithaca College: Master's.

3. American Psychological Association (2002). Ethical principles of psychologists and code of conduct. American Psychologist, 57(12), 10601073.

4. Anguera, M.T. (1992). Metodología de la observación en las ciencias humanas. Madrid: Cátedra.

5. Anguera, M.T., Arnau, J., Ato, M., Martínez, R., Pascual, J., y Vallejo, G. (1995). Métodos de investigación en psicología. Madrid: Síntesis.

6. Anguera, M.T., y Hernández-Mendo, A. (2013). La metodología observacional en el ámbito del deporte. e-balonmano.com: Revista de Ciencias del Deporte, 9(3), 135-161.
7. Anguera, M.T., y Hernández-Mendo, A. (2014). Metodología observacional y psicología del deporte: Estado de la cuestión. Revista de Ciencias del Deporte, 23(1), 103-109.

8. Arboix-Alió, J., y Aguilera-Castells, J. (2018). Influencia de marcar primero en hockey sobre patines. Cuadernos de Psicología del Deporte, 18(3), 220-231.

9. Arboix-Alió, J., y Aguilera-Castells, J. (2019). Analysis of the home advantage in roller hockey. Journal of Sport and Health Research, 3(11), 263-272.

10. Arboix-Alió, J., Aguilera-Castells, J., Buscà, B., Sánchez-López, M., y García-Eroles, L. (2019). Influence of half time score, match location and scoring first on match outcome in roller hockey. In 24th Annual Congress of the European College of Sport Science (pp. 417-418). Prague. doi:10.13140/RG.2.2.24509.82402

11. Arboix-Alió, J., Buscà, B., Trabal, G., AguileraCastells, J. y Sánchez-López, M. (2020). Comparison of home advantage in men's and women's Portuguese roller hockey league. Cuadernos de Psicología del Deporte, 20(1), 181-189.

12. Baumeister, R. F., y Steinhilber, A. (1984). Paradoxical effects of supportive audiences on performance under pressure: The home field disadvantage in sports championships. Journal of Personality and Social Psychology, 47, 85-93. doi:10.1037/0022-3514.47.1.85

13. Blanco-Villaseñor, A. y Escolano-Pérez, E. (2017). Observational data analysis using generalizability theory and general and mixed linear models: an empirical study of infant learning and development. Anales de Psicología, $33(3)$, 450-460. doi:10.6018/analesps.33.3.271021

14. Blanco-Villaseñor, A., Castellano, J., HernándezMendo, A., Sánchez-López, C. R. y Usabiaga, O. (2014). Aplicación de la TG en el deporte para el estudio de la fiabilidad, validez y estimación de la muestra. Revista de Psicología del Deporte, 23(1), 131-137

15. Blanco-Villaseñor, A., Castellano, J., HernándezMendo, A., Sánchez-López, C. R. y Usabiaga, O. (2014). Aplicación de la TG en el deporte para el 


\section{Variables contextuales para el portero de hockey patines faltas directas}

estudio de la fiabilidad, validez y estimación de la muestra. Revista de Psicología del Deporte, 23(1), 131-137.

16. Buceta, J.M. (1992). Intervención psicológica con el equipo nacional olímpico de baloncesto femenino. Revista de Psicología del Deporte, 2, 70-87.

18. Casimiro, E. (2010). Efeitos do local do jogo, da qualidade das equipas e dos períodos do jogo na performance do guarda-redes de Andebol. (Tesi de Licenciatura). Universidade de Trás-osMontes e Alto Douro, Vila Real.

19. Castellano, J., y Zubillaga, A. (1995a). Análisis de los goles Mundial de USA '94. El Entrenador Español, 64, 53-58.

20. Castellano, J., y Zubillaga, A. (1995b). Análisis de los goles Mundial de USA '94. El Entrenador Español, 65, 46-57.

21. Castellano, J., y Zubillaga, A. (1995c). Análisis de los goles Mundial USA. '94. El Entrenador Español, 66, 12-21.

22. Courneya, K., y Carron, A. (1992). The Home Advantage in Sport Competitions: A literature review. Journal of Sport \& Exercise Psychology, 14, 13-27. doi:10.1123/jsep.14.1.13

23. Cronbach, L.J., Gleser, G.C., Nanda, H. y Rajaratnam, N. (1972). The dependability of behavioral measurements: Theory of generalizability for scores and profiles. New York: Jhon Wiley and Sons.

24. Folguera, C. (2000). Portería a cero. El entrenamiento del portero de hockey sobre patines. Barcelona: Carles Folguera.

25. Gayton, W.F., Perry, S.M., Loignon, A.C., y Ricker, A. (2011). Re-examining the Home Disadvantage in Professional Ice Hockey. Perceptual and Motor Skills, 112, 600602. doi:10.2466/05.07.pms.112.2.600-602

26. Gayton, W.F., Steinroeder, W., Bonnica, C., y Loignon, A.C. (2009). An investigation of Home Disadvantage in Davis Cup Tennis. Perceptual and Motor Skills, 109, 382-386. doi:10.2466/pms.109.2.382-386

27. Gayton, W. F., Theriault, L., y Morneau, P. (2013). An Investigation of Home Disadvantage
17. Carron, A. V., Loughhead, T., y Bray, S. (2005). The Home Advantage in Sport Competitions: Courneya and Carron's (1992) conceptual framework a decade later. Journal of Sports Sciences, 23, 395-407. doi:10.1080/02640410400021542

in Fed Cup Tennis. Journal of Sport Behavior, 36(3), 257-263.

28. Gómez, M., Lago-Peñas, C., Viaño, J., y González-Garcia, I. (2014). Effects of Game Location, Team Quality and Final Outcome on Game-related Statistics in Professional Handball close games. Kineziologija, 46(2), 249-257.

29. Gómez, M., y Pollard, R. (2011). Reduced Home Advantage for Basketball Teams from Capital Cities in Europe. European Journal of Sport Science, 11(2). 143-148. doi:10.1080/17461391.2010.499970

30. Gómez, M., Pollard, R., y Luis-Pascual, J. C. (2011). Comparison of the home advantage in nine different professional team sports in Spain. Perceptual and Motor Skills, 113(1), 150-156. doi:10.2466/05.PMS.113.4.150-156

31. Gutiérrez, Ó., Saavedra, M., y FernándezRomero, J. (2015). Efecto de organizar un campeonato del mundo de balonmano en el rendimiento del equipo. Revista Internacional de Medicina y Ciencias de la Actividad Física y el Deporte, 15(57), 139-150. doi:10.15366/rimcafd2015.57.009

32. Hantau, C., y Hantau, C. (2014). Study Concerning the Effectiveness of Handball Goalkeeper at the $7 \mathrm{~m}$ Throws. Marathon, 6(1), 27-31.

33. Hernández Mendo, A. y Anguera, M. T. (2002). Behavioral Structure in Sociomotor Sports: Roller-Hockey. Quality \& Quantity, 36, 347-378

34. Hernández-Mendo, A., Blanco-Villaseñor, A., Pastrana, J. L., Morales-Sánchez, V., y RamosPérez, F. J. (2016). SAGT: Aplicación informática para análisis de generalizabilidad. Revista Iberoamericana de Psicología del Ejercicio y el Deporte, 11(1), 7789 


\section{Trabal, G., Daza, G. y Arboix-Alió, J. (2020)}

35. Hoffmann, M., Loughead, T., Dixon, J., y Crozier, A. (2017). Examining the Home Advantage in the National Hockey League: Comparisons Among Regulation, Overtime, and the Shootout. Psychology of Sport and Exercise, 28, 24-30. doi:10.1016/j.psychsport.2016.09.007

36. Ibañez, S., García, J., Feu, S., Parejo, I., y Canadas, M. (2009). La eficacia del tiro a canasta en la NBA: análisis multifactorial. Revista Cultura Ciencia y Deporte, 4, 39-47. doi:10.12800/ccd.v4i10.132

37. Jiménez-Torres, M., y López-Gutiérrez, C. (2012). El acierto en el tiro libre en baloncesto: cómo influye el minuto de partido, el estado del marcador y ser equipo local o visitante. Cuadernos de Psicología del Deporte, 12(2), 25-38. doi:10.4321/s157884232012000200004

38. Johnston, R. (2008). On Referee Bias, Crowd Size, and Home Advantage in the English Soccer Premiership. Journal of Sports Sciences, 26(6), 563 - 568. doi:10.1080/02640410701736780

39. Kozar, B., Vaughn, R. E., Whitfield, K. E., Lord, R. H., y Dye, B. (1994). Importance of FreeThrows at Various Stages of Basketball Games. Perceptual and Motor Skills, 78(1), 243-248. doi:10.2466/pms.1994.78.1.243

40. Labrador, F. J., Crespo, M., Buceta, J. M., y González, S. (1995). Factores contextuales implicados en el lanzamiento de tiros libres en baloncesto. Revista de Psicología del Deporte, 8, 87-99.

41. Legaz, A., Moliner, D., y Munguía, D. (2013). Home Advantage and Sports Performance: Evidence, Causes and Psychological Implications. Universitas Psychologica, 12(3), 933-943. doi:10.11144/javeriana.upsy12-3.hasp

42. Liardi, V.L., y Carron, A. V. (2011). An Analysis of National Hockey League Face-offs: Implications for the Home Advantage. International Journal of Sport and Exercise Psychology, 9(2), 102-109. doi: $10.1080 / 1612197 x .2011 .567100$

43. Loignon, A., Gayton, W.F., Brown, M., Steinroeder, W., y Johnson, C. (2007). Home Disadvantage in Professional Ice Hockey.
Perceptual and Motor Skills, 104, 1262-1264. doi:10.2466/pms.104.4.1262-1264

44. Maneiro, R. (2014). Análisis de las acciones a balón parado en el fútbol de alto rendimiento: saques de esquina y tiros libres indirectos: un intento de identificación de variables explicativas (Tesis doctoral). Universidade da Coruña, A Coruña.

45. McEwan, D., Ginis, K. A. M., y Bray, S. R. (2012). "With the Game on His Stick": The Home (dis)advantage in National Hockey League Shootouts. Psychology of Sport and Exercise, 13(5), 578-581. doi:10.1016/j.psychsport.2012.03.007

46. Morillo-Baro, J. P., Reigal, R. E., y HernándezMendo, A. (2015). Análisis del ataque posicional de balonmano playa masculino y femenino mediante coordenadas polares. RICYDE. Revista Internacional de Ciencias Del Deporte, 11(41), 226-244. doi:10.5232/ricyde2015.04103

47. Myers, T.D. (2014). Achieving external validity in home advantage research: generalizing crowd noise effects. Frontiers in Psychology, 5, 1-7. doi:10.3389/fpsyg.2014.00532

48. Myers, T.D. (2012). The impact of crowd noise on officiating in MuayThai: Achieving external validity in an experimental setting. Frontiers in Psychology, 3, 1-7. doi:10.3389/fpsyg.2012.00346

49. Navarro, B., Gómez, M., Lorenzo, J., Lorenzo, A., y Jiménez, S. (2015). La influencia del "home advantage" en el resultado de los momentos críticos en los partidos de baloncesto. Revista española de educación física y deportes, 396(229), 35-49.

50. Navia, J.A., y Ruiz, L.M. (2014). Análisis de la complejidad perceptivo-motriz y psicológica del penalti en el fútbol. Revista Internacional de Ciencias del Deporte, 10(37), 264-280. doi: 10.5232/ricyde

51. Nevill, A. M., y Holder, R. L. (1999). Home Advantage in Sport. Sports Medicine, 28(4), 221236. doi:10.2165/00007256-199928040-00001

52. Pauls, J. (2012). Táctica III: Alto rendimiento. Curso nacional de entrenadores de hockey 


\section{Variables contextuales para el portero de hockey patines faltas directas}

patines (Material no publicado). Barcelona: Real Federación Española de Patinaje.

53. Pollard, R. (1986). Home Advantage in Soccer: A Retrospective Analysis. Journal of Sports Sciences, $\quad 4$ 237-248. doi:10.1080/02640418608732122

54. Pollard, R. (2006). Home Advantage in soccer: Variations in its Magnitude and a Literature Review of the Inter-related Factors Associated with its Existence. Journal of Sport Behavior, 29, $169-89$.

55. Pollard, R., y Gómez, M. A. (2009). Home Advantage in Football in South-West Europe: Long-term Trends, Regional Variation, and Team Differences. Journal of Sports Sciences, 9(6), 341-352. doi:10.1080/17461390903009133

56. Rink Hockey Committee (CERH). (2009). Reglas del juego. Lisboa: CERH.

57. Roffé, M., De la Vega, R., Garcia-Mas, A. y Llinás, J. (2007). Las crisis durante el juego: el "gol psicológico" en el fútbol. Revista de Psicología del Deporte, 16(2), 227-240.

58. Sánchez, A., González, E., Ruiz, M., San-Juan, M., Abando, J., De Nicolás, L., y García, F. (2001). Estados de ánimo y rendimiento Deportivo en futbol: ¿existe la ventaja de jugar en casa? Revista de Psicología del Deporte, 10(2), 197-209.

59. Schlenker, B. R., Phillips, S. T., Boniecki, K. A., y Schlenker, D. R. (1995). Championship Pressures: Choking or Triumphing in One's Own Territory? Journal of Personality and Social Psychology, 68, 632-643. doi:10.1037/00223514.68.4.632

60. Smith, R. (2005). Disconnects Between Popular Discourse and Home Advantage Research: What Can Fans and Media Tell us About the Home Advantage Phenomenon? Journal of Sports Sciences, 23(4), 351-364. doi:10.1080/02640410400021633

61. Tauer, J. M., Guenther, C. L., y Rozek, C. (2009). Is there a Home Choke in Decisive Playoff Basketball Games? Journal of Applied Sport Psychology, 21, 148-162. doi:10.1080/10413200902795331
62. Trabal, G. (2016). Estudi etnogràfic del porter d'hoquei sobre patins: una vida entre paradoxes. Apunts. Educació física i esports, 126(4), 23-29. doi:10.5672/apunts.2014-

0983.es.(2016/4).126.02

63. Trabal, G. (2019). El portero de hockey patines. De la iniciación al alto rendimiento. Almería: Círculo rojo.

64. Trabal, G., Daza, G., y Riera, J. (2019a). Habilidades técnicas del portero de hockey patines. Retos, 36, 69-73.

65. Trabal, G., Daza, G., y Riera, J. (2019b). Influencia de las faltas directas en la clasificación OK Liga de hockey patines. Kronos, 18(1), 1-9.

66. Trabal, G., Daza, G., y Riera, J. (2020). La eficacia del portero en la falta directa del hockey patines. Apunts. Educación Física y Deportes, 139(1), 56-64. doi:10.5672/apunts.20140983.es.(2020/1).139.08

67. Vaz, V. (2011). Especialização desportiva em jovens hoquistas masculinos. Estudo do jovem atleta, do processo de selecção e da estrutura do rendimento (Tesis doctoral). Universitat de Coimbra, Coimbra.

68. Vázquez-Diz, J. A., Morillo-Baro, J. P., Reigal, R. E., Morales-Sánchez, V., \& HernándezMendo, A. (2019). Contextual Factors and Decision-Making in the Behavior of Finalization in the Positional Attack in Beach Handball: Differences by Gender Through Polar Coordinates Analysis. Frontiers in Psychology, 10. doi:10.3389/fpsyg.2019.01386

69. Whitehead, R., Butz, J. W., Kozar, B., y Vaughn, R. E. (1996). Stress and Perfomance: An Application of Gray's Three-factor Arousal Theory to Basketball Free Throw Shooting. Journal os Sport Science, 14, 393-401. doi:10.1080/026404196367697

70. Wilson, M.R., Vine, S.J., y Wood, G (2009). The influence of anxiety to visual attentional control in basketball free throw. Journal of Sport Exercise Psychology, 31, 152-168. doi:10.1123/jsep.31.2.152 